Revealing stacking sequences in inverse opals by microradian X-ray diffraction

This article has been downloaded from IOPscience. Please scroll down to see the full text article.

2010 EPL 8914002

(http://iopscience.iop.org/0295-5075/89/1/14002)

View the table of contents for this issue, or go to the journal homepage for more

Download details:

IP Address: 131.211.105.231

The article was downloaded on 26/01/2011 at 13:50

Please note that terms and conditions apply. 


\title{
Revealing stacking sequences in inverse opals by microradian X-ray diffraction
}

\author{
A. Sinitskit ${ }^{1(a)}$, V. Abramova ${ }^{1}$, N. Grigorieva ${ }^{2}$, S. Grigoriev ${ }^{3}$, A. Snigirev 4 , D. V. Byelov ${ }^{5}$ \\ and A. V. PETUKhov 5 \\ ${ }^{1}$ Department of Materials Science, M.V. Lomonosov Moscow State University - 119991 Moscow, Russia \\ ${ }^{2}$ Saint-Petersburg State University - 198504 Saint-Petersburg, Russia \\ ${ }^{3}$ Petersburg Nuclear Physics Institute - Gatchina, 188300 Saint-Petersburg, Russia \\ ${ }^{4}$ European Synchrotron Radiation Facility - BP 220, F-38043 Grenoble Cedex, France, EU \\ ${ }^{5}$ Debye Institute, Utrecht University - $3584 \mathrm{CH}$ Utrecht, The Netherlands, EU
}

received 30 September 2009; accepted in final form 10 December 2009

published online 22 January 2010

PACS 42.70.Qs - Photonic bandgap materials

PACS 41.60.Ap - Synchrotron radiation

PACS 61.05.cf - X-ray scattering (including small-angle scattering)

\begin{abstract}
We present the results of the structural analysis of inverse opal photonic crystals by microradian X-ray diffraction. Inverse opals based on different oxide materials $\left(\mathrm{TiO}_{2}, \mathrm{SiO}_{2}\right.$ and $\mathrm{Fe}_{2} \mathrm{O}_{3}$ ) were fabricated by templating polystyrene colloidal crystal films grown by the vertical deposition technique. Our results suggest that most inverse opal films possess dominating twinned face-centered cubic structure accompanied by some fragments of hexagonal close-packed and random hexagonal close-packed structures. The studied samples possessed individual structures with different ratios of the above fragments. By fitting the results of the angular-dependent X-ray diffraction by the Wilson model we estimate the stacking probability $\alpha$ in the studied samples to be $\sim 0.7-0.8$. Microradian X-ray diffraction therefore provides detailed structural information on opal-based photonic crystals and can be applied to opaque inverse opals or the samples with a periodicity $<300 \mathrm{~nm}$, whose structure cannot be investigated by conventional optical methods.
\end{abstract}

Copyright (C) EPLA, 2010

Introduction. - Over the last decade colloidal photonic crystals (PCs) are considered very promising for optoelectronics and photonics applications, since they can be fabricated at low cost, on a large scale, and both for visible and infrared spectral regions [1]. They can also serve as a suitable platform for the fabrication of inverse opal PCs based on high-refractive-index materials such as $\mathrm{Si}, \mathrm{Ge}, \mathrm{Sb}_{2} \mathrm{~S}_{3}, \mathrm{TiO}_{2}$, etc. $[2,3]$. According to the theoretical calculations, inverse opal with a face-centered cubic (fcc) structure and a refractive index contrast $\geqslant 2.8$ can possess a full photonic bandgap, a spectral range, for which the propagation of photons inside PC is completely inhibited for all directions [4]. However, inverse opals substantially inherit their structure from colloidal crystals, which in part typically have a close-packed, but not necessarily fcc structure. In general, a self-assembled colloidal crystal is composed of the close-packed layers, which can form

\footnotetext{
(a) Present address: Department of Chemistry, Rice University 6100 Main Street, Houston, TX 77005, USA; E-mail: alexander.sinitskiy@rice.edu
}

different structures depending on their stacking sequence: fcc structure (ABCABC...), hexagonal close-packed (hcp) structure (ABABAB...) as well as random hexagonal close-packed (rhcp) structure, for which close-packed layers are arranged in a random fashion [5]. Despite a calculated thermodynamic preference of fcc structure over hcp one due to a tiny entropy difference between these two structures [6], and numerous experimental evidences for preferable fcc crystallization of colloidal particles (see, for instance $[7,8]$ ), typical colloidal crystals are composed of fcc fragments separated by stacking faults or hcp fragments $[7,9,10]$.

Opals and inverse opals are widely used for different optical studies, in which the measurements are often performed on relatively small $(\sim 100 \mu \mathrm{m}$ in diameter $)$ single-domain areas of the samples in order to mitigate the effects of grain boundaries and other structural defects $[2,11,12]$. However, it is important to stress, that due to the random distribution of the stacking faults and hcp fragments throughout the sample, a finite-size opal or inverse opal domain consisted of a limited number 
of layers can, in principle, have any of the above structures, ranging from a pure fcc to rhcp or even hcp. Therefore, since each inverse opal sample is unique due to the structural defects, a method for the local analysis of the structure of self-assembled materials is highly demanded but remains a challenge. As will be shown here, conventional scanning electron microscopy (SEM) is difficult to be applied to the quantitative analysis of the stacking in inverse opals. Different optical methods, such as laser diffraction [13,14], confocal optical microscopy [15] and optical spectroscopy $[16,17]$ can only be applied to the optically transparent samples with a relatively large lattice constant ( several hundred $\mathrm{nm}$ ), which excludes the inverse opals for visible range with periods less that $300 \mathrm{~nm}[12,18-22]$. Furthermore, numerous inverse opals are fabricated from highly-absorbing materials, including carbon [23], transition metal oxides [24,25] and metals [26,27], and can thus be hardly studied by optical methods. Some advanced imaging techniques, such as high-resolution X-ray microscopy or SEM combined with the focused ion beam technique, could also be potentially employed for the structural studies of inverse opals. However, these methods can only be utilized for the local analysis, whereas the integral structural information about inverse opal domains, suitable for the optical measurements, is often required.

Here we report on the study of fragments with different stacking sequences in inverse opals by microradian X-ray diffraction. Previously, X-ray diffraction was successfully applied to colloidal crystals [28-35], but rarely used for the study of inverse opals [36]. The samples used for this study were fabricated from different oxide materials (including highly-absorbing ferric oxide, $\mathrm{Fe}_{2} \mathrm{O}_{3}$ ), had a periodicity less than $300 \mathrm{~nm}$, and thus practically could not be analyzed by conventional methods for the structural analysis.

Experimental section. - All chemicals were purchased from Aldrich unless otherwise specified. Monodisperse polystyrene microspheres with an average diameter of $420 \mathrm{~nm}$ and a relative standard deviation $\sigma<5 \%$ were synthesized by emulsifier-free emulsion polymerization of styrene using potassium persulfate as initiator [37]. Colloidal crystal films were grown on glass microslides at $50 \pm 1{ }^{\circ} \mathrm{C}$ from $\sim 1$ vol. $\%$ aqueous suspension of microspheres by the vertical deposition method [38].

The samples discussed in this paper are inverse opals based on $\mathrm{SiO}_{2}, \mathrm{TiO}_{2}$ and $\mathrm{Fe}_{2} \mathrm{O}_{3}$ (8 samples total). Inverse opals based on iron oxide were synthesized by infiltrating colloidal crystal templates with a water-alcohol $(1: 1)$ solution of iron (III) nitrate, drying the samples in air, heating the resulting composites at the rate of $1^{\circ} \mathrm{C} / \mathrm{min}$ up to $500^{\circ} \mathrm{C}$, and annealing for $1 \mathrm{~h}$. Silica inverse opals were prepared by infiltrating colloidal crystals with acidic solution of tetraethyl orthosilicate (TEOS, 98\%) (43 vol.\% TEOS - 29 vol. $\% \mathrm{C}_{2} \mathrm{H}_{5} \mathrm{OH}-21$ vol. $\% \mathrm{H}_{2} \mathrm{O}-7$ vol. $\%$ $\mathrm{HCl}$ ), drying the samples in air, heating them up to $500{ }^{\circ} \mathrm{C}$ $\left(1^{\circ} \mathrm{C} / \mathrm{min}\right)$, and annealing for $1 \mathrm{~h}$. For the fabrication

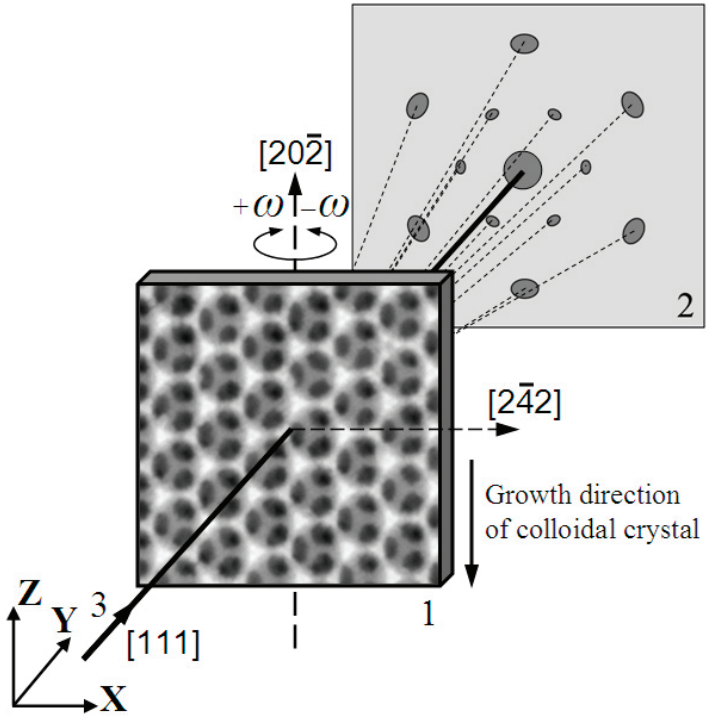

Fig. 1: Schematic of the microradian X-ray diffraction experiment: 1) sample, 2) two-dimensional CCD detector, 3) X-ray beam. The samples were mounted on the goniometer perpendicularly to the X-ray beam the same way as during colloidal crystal growth; the Miller indices for the characteristic directions are shown as if the sample had an ideal $\mathrm{fcc}_{1}$ structure. Here and later the same reference frame is shown.

of titania inverse opals we prepared a dipping solution by diluting titanium (IV) butoxide with heptane (Acros Organics, $99+\%)$ in a $1: 1$ ratio. Colloidal crystal films were infiltrated with the dipping solution, dried in air, heated up to $500^{\circ} \mathrm{C}$ at the rate of $1^{\circ} \mathrm{C} / \mathrm{min}$ and annealed for $1 \mathrm{~h}$. According to the results of X-ray powder diffraction (XPD), iron oxide inverse opal consisted of crystalline hematite (PDF-2 file [33-664]), XPD pattern of titanium oxide inverse opal was attributed to crystalline anatase (PDF-2 file [21-1272]), whereas silica in the synthesized inverse opal samples was amorphous. The microstructure of inverse opals was studied by SEM using a LEO Supra VP 35 instrument. No conductive coating was applied to the samples prior to imaging.

Microradian X-ray diffraction experiments were performed at DUBBLE BM-26 line at the European Synchrotron Radiation Facility (Grenoble, France). The details on the experimental setup are given in $[15,28]$. Briefly, diffraction of a monochromatic $13 \mathrm{keV}$ X-ray beam $\left(\lambda=0.095 \mathrm{~nm} ; \Delta \lambda / \lambda=2 \cdot 10^{-4}\right)$ was registered behind the sample at the distance of $8 \mathrm{~m}$ by a two-dimensional charge-coupled device (CCD) detector (Photonic Science) with $4008 \times 2671$ pixels of $22.7 \mu \mathrm{m} \times 22.7 \mu \mathrm{m}$. In order to achieve angular resolution needed to study these largeperiod structures, the beam was focused at the detector by a set of compound refractive lenses [39]. The diameter of the X-ray beam was about $0.5 \mathrm{~mm}$. Figure 1 shows the schematic of the experimental setup. The samples were mounted on the goniometer perpendicularly to the X-ray beam the same way as during colloidal crystal growth. First, we scanned the samples in $X Z$-plane to find the areas exhibiting only six distinct diffraction spots [14]. 


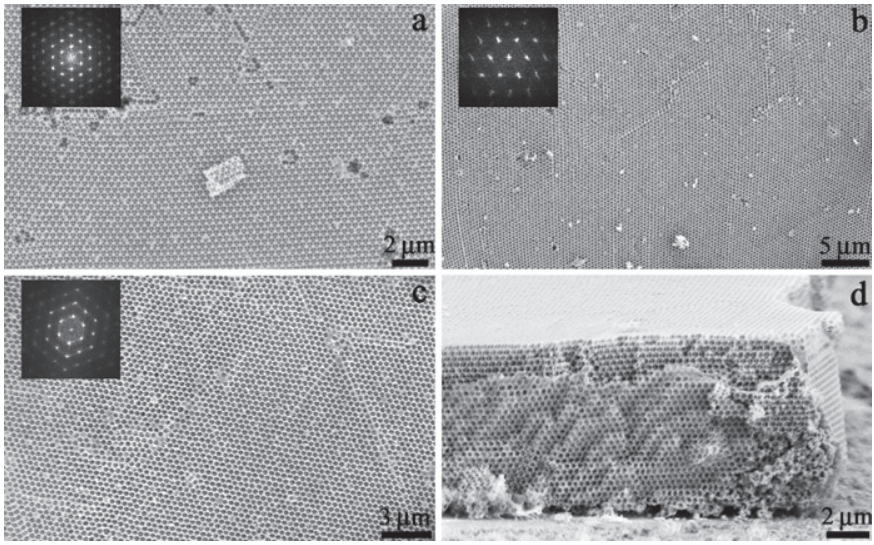

Fig. 2: SEM images of (a) $\mathrm{Fe}_{2} \mathrm{O}_{3}$, (b) $\mathrm{TiO}_{2}$ and (c) $\mathrm{SiO}_{2}$ inverse opals. The Fourier transforms of the images are shown in the insets. (d) Cross-sectional SEM image of $\mathrm{TiO}_{2}$ inverse opal.

Then, samples were rotated around the vertical axis, and the diffraction patterns were recorded at different angles of rotation $\omega$, typically from $-60^{\circ}$ to $+60^{\circ}$, while $\omega=0^{\circ}$ corresponded to the normal incidence of the X-ray beam on the sample surface.

Results and discussion. - According to the results of SEM, the average center-to-center distances between spherical voids are $390 \mathrm{~nm}$ for $\mathrm{TiO}_{2}$ inverse opals, $380 \mathrm{~nm}$ for $\mathrm{Fe}_{2} \mathrm{O}_{3}$ inverse opals and $365 \mathrm{~nm}$ for $\mathrm{SiO}_{2}$ inverse opals, which are less than the average size of the polystyrene microspheres used for the template preparation $(420 \mathrm{~nm})$. This effect is attributed to the shrinkage of the sample due to removal of volatile components during annealing $[14,40]$. Thus, our inverse opal films consisted of ordered domains separated by cracks. Figures $2 \mathrm{a}-\mathrm{c}$ show typical SEM images of the domains in three different oxide inverse opals. Typical domain size was $25-500 \mu \mathrm{m}^{2}$, though some individual domains could be $>2500 \mu \mathrm{m}^{2}$ in size (fig. $2 \mathrm{~b}$ ). Obviously, such SEM images can be used to verify the ordering in the top layer of the sample but give no information on whether the structure of inverse opal is fcc or hcp or another one. One can expect to obtain such information from the cross-sectional SEM images of the samples. However, it can be clearly seen in a typical cross-sectional SEM image of one of the titania inverse opal films (fig. 2d), that the sample consists of 31 layers, but their stacking sequence is difficult to determine. In fact, none of crosssectional SEM image recorded for the cleaved surfaces of inverse opals was descriptive enough to provide the information about the stacking sequences in these samples.

In this paper we demonstrate that structural information for inverse opals can be extracted from the microradian X-ray diffraction patterns. We have investigated different areas of inverse opals, which exhibited only six distinct diffraction spots without an apparent diffuse ring; such areas were found by the in-plane scanning of the samples with the X-ray beam, as described in ref. [14]. Obviously, since the results of SEM suggest that the typical domain size was $25-500 \mu \mathrm{m}^{2}$, i.e. much less than the

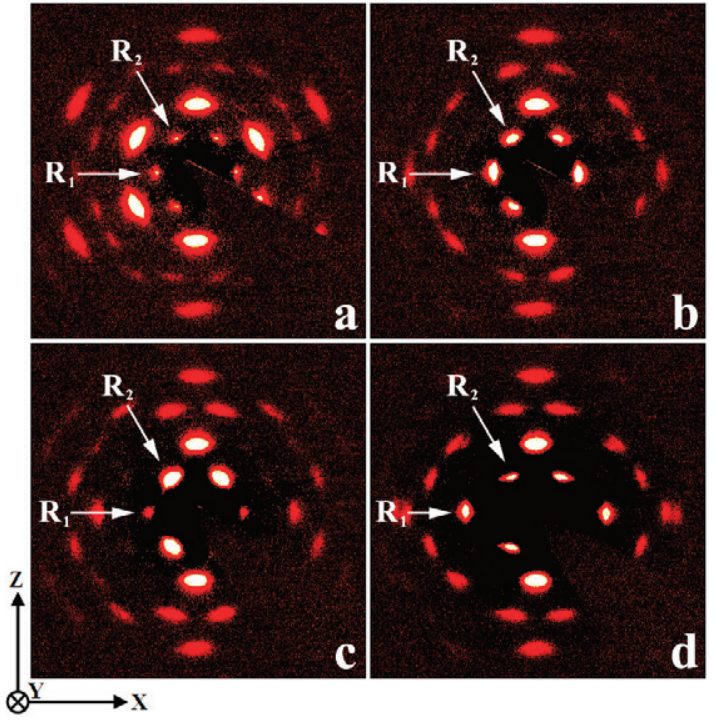

Fig. 3: (Colour on-line) Microradian X-ray diffraction patterns recorded for $\mathrm{Fe}_{2} \mathrm{O}_{3}$ inverse opal at different angles of incidence $\omega:\left(\right.$ a) $0^{\circ}$, (b) $+19.5^{\circ}$, (c) $+35.3^{\circ}$, and (d) $+54.7^{\circ}$.

beam size, it was most likely that a few domains rather than an individual giant domain were irradiated by the X-ray beam. However, since these domains gave clear sixfold diffraction patterns, they had nearly the same crystallographic orientation and thus could be effectively considered as one single-domain region [14]. Interestingly, it was possible to find such areas of about $0.5 \mathrm{~mm}$ in diameter (the size of the X-ray beam) in all our inverse opal samples despite their inevitable cracking during the synthesis $[14,40]$. The presence of cracks and domains with different orientation makes inverse opals more difficult objects for the structural analysis than the original colloidal crystals.

Figure 3 shows the sequential diffraction patterns recorded for the selected area of $\mathrm{Fe}_{2} \mathrm{O}_{3}$ inverse opal at different angles of rotation $\omega$. At normal incidence bright spots corresponding to several orders of diffraction are clearly seen (fig. 3a). The brightest spots can be attributed to X-ray diffraction on the crystallographic planes in a close-packed structure. Weaker reflections in fig. 3a (such as those marked as $R_{1}$ and $R_{2}$ ) can be attributed to either finite thickness of the crystal or stacking disorder as well as other types of disorder [41]. The variations of intensities of the $R_{1}$ and $R_{2}$ reflections upon the sample rotation can be followed in fig. 3 . The first spot has a relatively low intensity at $\omega=0^{\circ}$, then it reaches intensity maximum at $\omega=+19.5^{\circ}$, gets low intensity at $\omega=+35.3^{\circ}$ and then reaches the second intensity maximum at $\omega=+54.7^{\circ}$. Apparently, the position of $\mathrm{R}_{1}$ spot slightly drifts outward the center of the diffraction pattern along the horizontal axis. The displacement of $\mathrm{R}_{2}$ spot in the diffraction pattern is less obvious, since this spot is closer to the rotation axis; the maximum of intensity is observed at $\omega=+35.3^{\circ}$. The behaviors of $R_{1}$ and $R_{2}$ spots in the diffraction patterns recorded for 


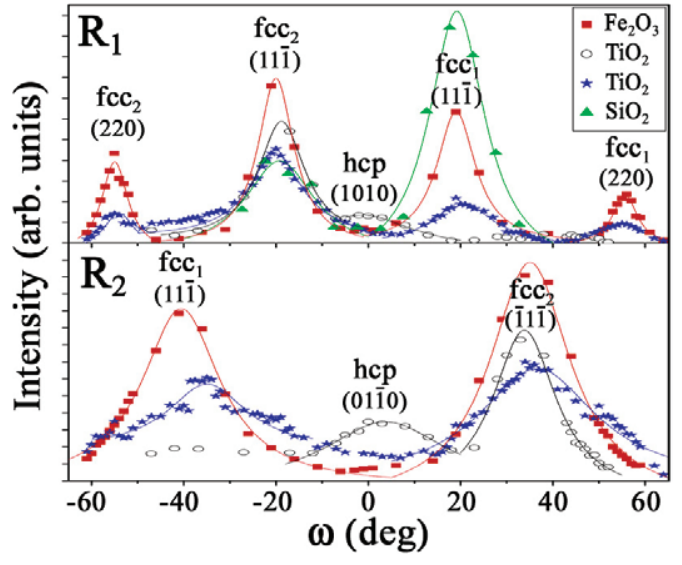

Fig. 4: (Colour on-line) Intensities of diffraction spots (a) $R_{1}$ and (b) $\mathrm{R}_{2}$ as functions of the incidence angle $\omega$ for selected $\mathrm{Fe}_{2} \mathrm{O}_{3}, \mathrm{SiO}_{2}$ and $\mathrm{TiO}_{2}$ inverse opals. Symbols correspond to the experimental data, whereas solid lines are their fittings with Lorentz functions.

other inverse opal samples were different but also with apparent maxima at specific angles. Angular intensity dependencies for $R_{1}$ and $R_{2}$ spots for the selected areas found in $\mathrm{Fe}_{2} \mathrm{O}_{3}, \mathrm{SiO}_{2}$ and two different $\mathrm{TiO}_{2}$ inverse opal samples are shown in fig. 4.

In order to explain the behavior of $R_{1}$ and $R_{2}$ spots we should consider the structures of possible fragments $\left(\mathrm{fcc}_{1}\right.$ (ABCABC...), $\mathrm{fcc}_{2}$ (ACBACB...) and hcp) of inverse opal samples in a reciprocal space. Figure 5 a shows the $\mathrm{fcc}_{1}$ structure in the reciprocal lattice along with the reference frame used in the diffraction experiments. We should observe a diffraction maximum if at some angle of incidence the Ewald sphere intersects corresponding nodes of the reciprocal lattice. Therefore, in order to index the diffraction maxima in the angular dependencies in fig. 4, we need to determine such angles for all possible structures (fcc $1, \mathrm{fcc}_{2}$ and hcp). Since $a \gg \lambda$, where $a$ is a period of inverse opal and $\lambda$ is the wavelength of a synchrotron radiation, the radius of the Ewald sphere $2 \pi / \lambda$ is more than three orders of magnitude larger than the characteristic distance $2 \pi / a$ for inverse opal lattice in a reciprocal space. Thus, we can neglect the curvature of the Ewald sphere and simply consider the cross-section of the reciprocal lattice with the Ewald sphere as a plane. Within the geometry of the experiment this "Ewald plane" is always perpendicular to the horizontal plane $(X Y)$, and therefore it is more convenient to consider the projections of characteristic reciprocal lattice points for $\mathrm{fcc}_{1}, \mathrm{fcc}_{2}$ and hcp structures onto the horizontal plane. These projections are shown in figs. $5 \mathrm{~b}-\mathrm{d}$.

In the case of the $\mathrm{fcc}_{1}$ structure, following the $\mathrm{R}_{1}$ spot in fig. 3 is equivalent to moving in a reciprocal space along the correspondingly marked dashed line in fig. 5b. Clearly, Ewald sphere intersects reciprocal lattice points on the dashed line " $\mathrm{R}_{1}$ " at $\omega=-35.3^{\circ},+19.5^{\circ}$ and $+54.7^{\circ}((00 \overline{2}),(11 \overline{1})$ and $(220)$ reflections, respectively), and thus for an inverse opal with pure fcc structure $_{1}$ we can expect to observe the intensity maxima in the

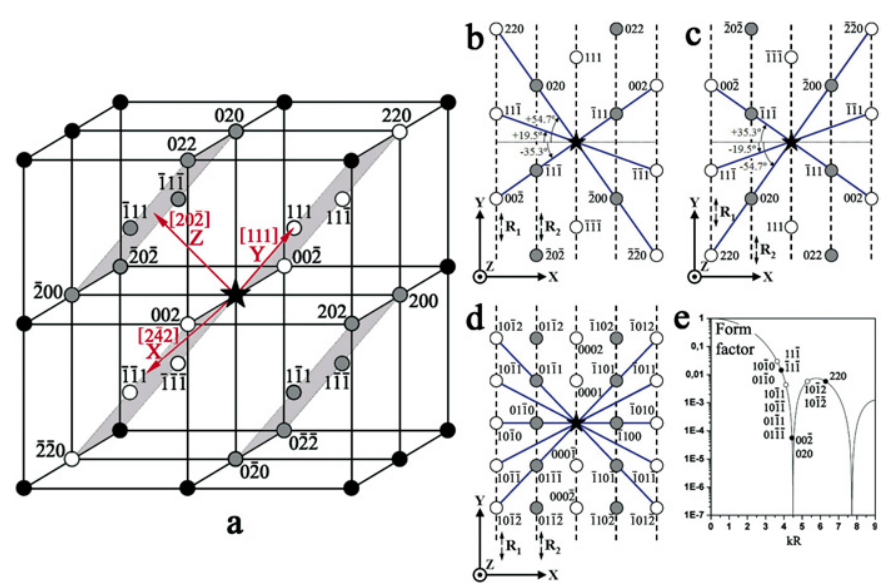

Fig. 5: (Colour on-line) The reciprocal lattice for the fcc f $_{1}$ structure (a) and the projections of characteristic reciprocal lattice points for (b) fcc 1 (ABCABC...), (c) fcc 2 (ACBACB...) and (d) hcp (ABABAB...) onto the horizontal plane $(X Y)$ within the geometry of the experiment. Note that the reciprocal lattice points, shown in gray and white circles in (b)-(d), belong to different planes in the corresponding 3D structures. For the $\mathrm{fcc}_{1}$ structure these planes are colored in light gray in (a); these planes are parallel and separated by $2 \pi / a$ spacing, where $a$ is a diameter of the spherical void in the inverse opal. In each panel the black star marks the origin of the reciprocal space. In panels (b)-(d) the solid lines show the intersections of the Ewald sphere with the reciprocal lattice points at certain angles of incidence $\omega$. (e) Theoretical form factor of a single sphere. Symbols show the form factor values for the different reciprocal lattice points shown in (b)-(d). Black dots correspond to the $\mathrm{fcc}_{1}$ and $\mathrm{fcc}_{2}$ structures; white spots correspond to the hcp structure.

angular dependence of the intensity of the $R_{1}$ diffraction spot at these angles only. Furthermore, these diffraction maxima should have different intensities as determined not only by the structure factor but also by the form factor of the individual scattering element (i.e., the sphere) $[30,32,42]$. Figure 5e shows that the form factor for the $(11 \overline{1})$ reflection is slightly higher than that for the (220) reflection, while both are two orders of magnitude higher than that for the $(00 \overline{2})$ reflection. Thus, in a real diffraction experiment for an inverse opal with pure fcc $_{1}$ structure we should observe only two maxima in the angular dependence of the $R_{1}$ spot: the most intense peak at $\omega=+19.5^{\circ}$ and the second peak at $\omega=+54.7^{\circ}$. The intensity maxima for other possible structures can be found in a similar way for both $R_{1}$ and $R_{2}$ diffraction spots (see table 1).

The data presented in table 1 allows for indexing intensity maxima in fig. 4 . We can conclude that the selected region of the $\mathrm{Fe}_{2} \mathrm{O}_{3}$ inverse opal sample discussed hereinabove had a twinned fcc structure composed by $\mathrm{fcc}_{1}$ and $\mathrm{fcc}_{2}$ fragments. By calculating the ratio of the peak areas for similar reflections, i.e. at $\omega=+19.5^{\circ}((11 \overline{1})$ in $\left.\mathrm{fcc}_{1}\right)$ and $-19.5^{\circ}\left((11 \overline{1})\right.$ in $\left.\mathrm{fcc}_{2}\right)$ we can estimate the ratio of $\mathrm{fcc}_{1}$ and $\mathrm{fcc}_{2}$ fragments in the sample to be $\sim 2: 3$. Noteworthy, twinned fcc structure was found for different regions in 7 out of 8 inverse opals, in which 
Table 1: Possible diffraction maxima for $R_{1}$ and $R_{2}$ spots in the range $-57^{\circ}<\alpha<+57^{\circ}$ for the basic structures: angles, Miller indices and relative intensities. Intensity of reflection: $\mathrm{VH}$ (very high), $\mathrm{H}$ (high), $\mathrm{N}$ (neglible).

\begin{tabular}{|c|c|c|c|}
\hline \multirow[t]{2}{*}{ Diffraction spot } & \multicolumn{3}{|c|}{ Structure } \\
\hline & $\mathrm{fcc}_{1}(\mathrm{ABCABC} \ldots)$ & $\mathrm{fcc}_{2}(\mathrm{ACBACB} \ldots)$ & hcp (ABABAB...) \\
\hline $\mathrm{R}_{1}$ & $\begin{array}{l}-35,3^{\circ},(00 \overline{2}), \mathrm{N} \\
+19,5^{\circ},(11 \overline{1}), \mathrm{VH} \\
+54,7^{\circ},(220), \mathrm{H}\end{array}$ & $\begin{array}{l}-54,7^{\circ},(220) \mathrm{H} \\
-19,5^{\circ},(11 \overline{1}) \mathrm{VH} \\
+35,3^{\circ},(00 \overline{2}) \mathrm{N}\end{array}$ & $\begin{array}{l}-46,7^{\circ},(10 \overline{1} \overline{2}) \mathrm{H} \\
-27,9^{\circ},(10 \overline{1} \overline{1}) \mathrm{H} \\
0^{\circ},(10 \overline{1} 0) \mathrm{VH} \\
+27,9^{\circ},(10 \overline{1} 1) \mathrm{H} \\
+46,7^{\circ},(10 \overline{1} 2) \mathrm{H}\end{array}$ \\
\hline $\mathrm{R}_{2}$ & $\begin{array}{l}-35,3^{\circ},(\overline{1} 1 \overline{1}) \mathrm{VH} \\
+54,7^{\circ},(020) \mathrm{N}\end{array}$ & $\begin{array}{l}-54,7^{\circ},(020) \mathrm{N} \\
+35,3^{\circ},(\overline{1} 1 \overline{1}) \mathrm{VH}\end{array}$ & $\begin{array}{l}-46,7^{\circ},(01 \overline{1} \overline{1}) \mathrm{H} \\
0^{\circ},(01 \overline{1} 0) \mathrm{VH} \\
+46,7^{\circ},(01 \overline{1} 1) \mathrm{H}\end{array}$ \\
\hline
\end{tabular}

the amounts of $\mathrm{fcc}_{1}$ and $\mathrm{fcc}_{2}$ could be fairly equal or one phase could dominate over another one. Only one sample exhibited weak diffraction peaks associated to the hcp structure (one of titania inverse opal samples had a predominant $\mathrm{fcc}_{2}$ structure with apparent hcp fragments; see the angular dependencies in fig. 4 shown as white circles). The dominant cubic arrangement of the air voids in inverse opals results in a clear square-like arrangement of the reflections in the diffraction patterns recorded at $\omega= \pm 54.7^{\circ}$ (fig. 3d). Since the structures of inverse opals are mostly determined by the structures of colloidal crystal templates, we consider this experimental fact as an evidence for the preferable fcc crystallization of colloidal microspheres during vertical deposition.

Obviously, in case of the ideal structures we would observe diffraction spots $R_{1}$ and $R_{2}$ only at specific angles discussed hereinabove. However, for all samples discussed the diffraction intensity is not zero at any angle $\omega$ neither for $R_{1}$ nor for $R_{2}$ diffraction spot, which can be explained by the finite thickness of the samples and the presence of stacking faults. Remarkably, structures with numerous stacking faults (close to rhcp) exhibit very broad diffraction peaks $[33,42,43]$. Since in our case diffraction peaks are sharp and observed almost at their calculated "ideal" fcc and hcp positions (fig. 4), we conclude that the density of stacking faults in these samples was quite low.

Though typically we have studied our samples in the range $-60^{\circ} \leqslant \omega \leqslant+60^{\circ}$, the most of the structural information could be obtained by recording angle-dependent diffraction patterns in the range $-30^{\circ} \leqslant \omega \leqslant+30^{\circ}$. As an example we show the angular dependence of the intensity of $R_{1}$ diffraction spot for the silica inverse opal sample (fig. 4). According to these data, the analyzed region of the sample had a twinned fcc structure composed by fcc $\mathrm{fc}_{1}$ and $\mathrm{fcc}_{2}$ fragments with a ratio of $\sim 4: 9$. Due to the high intensity of the synchrotron radiation and the limited $\omega$-range, such express structural analysis can be done in a matter of minutes.

The structure of self-assembled colloidal crystals is often characterized by the percentage $\alpha$ of randomly distributed fcc fragments in the sample. Correspondingly, for the ideal hcp structure $\alpha=0$, for the ideal fcc structure $\alpha=1$. For sedimentary rhcp crystals the value of $\alpha$ is often close to

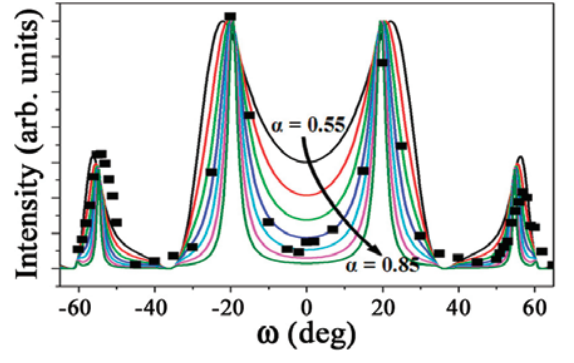

Fig. 6: (Colour on-line) Angular dependence of the intensity of the $\mathrm{R}_{1}$ diffraction spot for the selected area of the $\mathrm{Fe}_{2} \mathrm{O}_{3}$ inverse opal (black squares). The solid curves correspond to the results of the calculations by the Wilson model for $\alpha$ ranging from 0.55 to 0.85 with a step of 0.05 .

$0.5[33,34]$. For the samples investigated by microradian $\mathrm{X}$-ray diffraction $\alpha$ can be estimated by fitting the angular dependencies shown in fig. 4 by the Wilson model [44]. In this model it is assumed that the intensity of the scattered radiation $I(\mathbf{q})$ can be written as $I(\mathbf{q}) \propto S(\mathbf{q}) \cdot F(q)$, where $\mathbf{q}$ is the scattering vector, $S(\mathbf{q})$ is the structure factor and $F(q)$ is the form factor of a single building block of the structure. The calculation of $S(\mathbf{q})$ along the Bragg rods followed that described in $[42,44]$. For the calculation of $F(q)$ the inverse opals were assumed to consist of monodisperse uniform air microspheres touching each other.

As an example, we demonstrate in fig. 6 the comparison of experimental data for the intensity of the $\mathrm{R}_{1}$ reflection for the $\mathrm{Fe}_{2} \mathrm{O}_{3}$ inverse opal (the same angular dependence is shown as squares in fig. 4) with the results of the calculations by the Wilson model for $\alpha$ ranging from 0.55 to 0.85 . It is clear, that the best agreement with the experimental data is achieved for $\alpha=0.7-0.8$, which confirms our previous conclusion that the fcc structure is dominating over the sample, although a certain amount of stacking faults is present. The deviation of the fittings from the experimental data could be, in principle, attributed to several factors such as non-symmetry of the angular dependence due to the 2:3 ratio of $\mathrm{fcc}_{1}$ and $\mathrm{fcc}_{2}$ fragments in the sample, finite thickness of the inverse opal film, etc.

Noteworthy, the reported X-ray diffraction data were obtained for the areas of the inverse opal samples of about $0.2 \mathrm{~mm}^{2}$ in size, which was limited by the diameter 
of the X-ray beam. Therefore, the results of our work suggest that for a sub-millimeter area of inverse opal one can expect virtually any structure ranging from a typical twinned fcc structure (with a different ratio of $\mathrm{fcc}_{1}$ and $\mathrm{fcc}_{2}$ fragments) to the structure with only one dominating fcc phase. Importantly, such sample areas should exhibit different optical properties [10]. Therefore, extreme caution should be used for the interpretation of the results of optical measurements, which are often performed on the PC domains, which are less than $100 \cdot 100 \mu \mathrm{m}^{2}$ in size $[10,12,22]$. On the other hand, the microradian X-ray diffraction can also be used for finding the areas of self-assembled PCs with a desired structure.

Conclusions. - In conclusion, we presented a method for the structural analysis of self-assembled PCs based on microradian X-ray diffraction. The method was applied to inverse opals based on different oxide materials $\left(\mathrm{TiO}_{2}\right.$, $\mathrm{SiO}_{2}, \mathrm{Fe}_{2} \mathrm{O}_{3}$ ), for which the stacking sequences (the presence of $\mathrm{fcc}_{1}$, f $\mathrm{fcc}_{2}$, hcp and rhcp fragments) were estimated. In all samples studied we could find the areas of at least $0.5 \mathrm{~mm}$ in diameter, which exhibited only six distinct diffraction spots, indicating the presence of an individual giant domain or a few domains with nearly the same crystallographic orientation. Each of those areas was found to possess an individual structure, which should be taken into account in the optical studies of such samples. Our results suggest that thus-synthesized inverse opal films typically have twinned fcc structures (though they may also contain hcp fragments), in which the amounts of $\mathrm{fcc}_{1}$ and $\mathrm{fcc}_{2}$ phases could be fairly equal or one phase could dominate over the other one. By fitting the results of the angular X-ray diffraction by the Wilson model, we estimate the stacking probability $\alpha$ in the studied samples to be $\sim 0.7-0.8$. The described method of the structural analysis can also be applied to the study of inverse opals based on different materials, and, in general, to various self-assembled structures with submicron periodicity.

The work was supported by the Russian Foundation for Basic Research (grants Nos. 07-03-92113 and 08-03-00938) and the Federal Target Science and Engineering Program. We thank the Netherlands Organization for Scientific Research (NWO) for granting us the beam time and the personnel of the beamline BM-26 for their excellent support.

\section{REFERENCES}

[1] Xia Y., Gates B., Yin Y. and Lu Y., Adv. Mater., 12 (2000) 693.

[2] Blanco A. et al., Nature, 405 (2000) 437.

[3] López C., Adv. Mater., 15 (2003) 1679.

[4] Busch K. and John S., Phys. Rev. E, 58 (1998) 3896.
[5] Kittel C., Introduction to Solid State Physics, 7th edition (Wiley, New York) 1996.

[6] Woodcock L., Nature, 385 (1997) 141.

[7] Sanders J. V., Nature, 204 (1964) 1151.

[8] Miguez H. et al., Langmuir, 13 (1997) 6009.

[9] Pusey P. N. et al., Phys. Rev. Lett., 63 (1989) 2753.

[10] Vlasov Yu. A. et al., Phys. Rev. E, 61 (2000) 5784.

[11] Garcia P. D. and López C., J. Appl. Phys., 99 (2006) 046103.

[12] Li M. et al., Appl. Phys. B, 89 (2007) 251.

[13] Amos R. M. et al., Phys. Rev. E, 61 (2000) 2929.

[14] Sinitskit A., Abramova V., Laptinskaya T. and Tretyakov Y. D., Phys. Lett. A, 366 (2007) 516.

[15] Thijssen J. H. J. et al., Adv. Mater., 18 (2006) 1662.

[16] Checoury X., Enoch S., López C. and Blanco A., Appl. Phys. Lett., 90 (2007) 161131.

[17] Vekris E. et al., Adv. Mater., 20 (2008) 1110.

[18] Li B. et al., Appl. Phys. Lett., 82 (2003) 3617.

[19] Abramova V. and Sinitskil A., Superlattices Microstruct., 45 (2009) 624.

[20] Koenderink A. F. et al., Phys. Rev. Lett., 88 (2002) 143903.

[21] Solovyev V. G. et al., J. Appl. Phys., 94 (2003) 1205.

[22] Schriemer H. P., van Driel H. M., Koenderink A. F. and Vos W. L., Phys. Rev. A, 63 (2000) 011801.

[23] Zakhidov A. A. et al., Science, 282 (1998) 897.

[24] YAN H. et al., Adv. Mater., 11 (1999) 1003.

[25] YAN H. et al., Chem. Mater., 12 (2000) 1134.

[26] Bartlett P. N., Baumberg J. J., Coyle S. and Abdelsalam M. E., Faraday Discuss., 125 (2004) 117.

[27] Napolskit K. S. et al., Physica B, 397 (2007) 23.

[28] Petukhov A. V. et al., J. Appl. Crystallogr., 39 (2006) 137.

[29] Vos W. L., Megens M., van Kats C. M. and Bösecke P., Langmuir, 13 (1997) 6004.

[30] Megens M., van Kats C. M., Bösecke P. and Vos W. L., Langmuir, 13 (1997) 6120.

[31] Peтukhov A. V. et al., Phys. Rev. Lett., 88 (2002) 208301.

[32] Xian X. et al., J. Appl. Crystallogr., 36 (2003) 597.

[33] Peтukhov A. V. et al., Phys. Rev. Lett., 90 (2003) 028304.

[34] Dolbnya I. P. et al., Europhys. Lett., 72 (2005) 962.

[35] Hilhorst J. et al., Langmuir, 25 (2009) 10408.

[36] Wijnhoven J. E. G. J., Bechger L. and Vos W. L., Chem. Mater., 13 (2001) 4486.

[37] Goodwin J. W., Hearn J., Ho C. C. and Ottewill R. H., Colloid Polym. Sci., 252 (1974) 464.

[38] Jiang P., Bertone J. F., Hwang K. S. and Colvin V. L., Chem. Mater., 11 (1999) 2132.

[39] Snigirev A., Kohn V., Snigireva I. and Lengeler B., Nature, 384 (1996) 49.

[40] Sinitski A. et al., Superlattices Microstruct., 44 (2008) 626.

[41] Förster S. et al., Nat. Mater., 6 (2007) 888.

[42] Guinier A., X-ray Diffraction in Crystals, Imperfect Crystals and Amorphous Bodies (W.H. Freeman \& Co., San Francisco) 1963.

[43] Loose W. and Ackerson B. J., J. Chem. Phys., 101 (1994) 7211.

[44] Wilson A. J. C., X-ray Optics (Methuen \& Co. Ltd., London) 1949. 